\title{
Vitamins with anti-inflammatory properties in diabetic and nondiabetic subjects
}

\author{
A. Valente ${ }^{1}$, M. Bicho ${ }^{2,3}$, T. G. Albuquerque ${ }^{1}$, R. Duarte ${ }^{4}$, J. F. Raposo ${ }^{4}$ and H. S. Costa ${ }^{1}$ \\ ${ }^{1}$ Departamento de Alimentação e Nutrição (DAN), Instituto Nacional de Saúde Doutor Ricardo Jorge, I.P. (INSA), \\ Av. Padre Cruz, 1649-016 Lisboa, Portugal, ${ }^{2}$ Laboratório de Genética, Faculdade de Medicina da Universidade de Lisboa, \\ Av. Prof. Egas Moniz, 1649-028 Lisboa, Portugal, ${ }^{3}$ Instituto Rocha Cabral, Calçada Bento da Rocha Cabral, 14, 1250-047 \\ Lisboa, Portugal and ${ }^{4}$ Associação Protectora dos Diabéticos de Portugal (APDP), Rua do Salitre 118-120, 1250-203 \\ Lisboa, Portugal
}

Type 2 diabetes mellitus predisposes patients to increased susceptibility to various infections. Nutrition plays a critical role in maintaining normal immune function. Adequate status of antioxidant vitamins are required for the immune system to function efficiently. The aim of the study is to evaluate the blood status of the major antioxidant vitamins with anti-inflammatory proprieties in diabetic patients and nondiabetic subjects. The blood levels of vitamins C, A and E were measured in 150 Portuguese type 2 diabetic patients and 143 health age-matched controls using previously validated HPLC methods. The study population was divided into three groups: group I-75 diabetics with angiopathy; group II- 75 diabetics without angiopathy and group III-143 nondiabetic subjects. The statistical analysis was performed by one-way analysis of variance and Pearson Correlation Coefficient. Group III had a lower mean level of vitamin E ( $22.2 \mu \mathrm{M})$ compared to the mean values obtained for groups I $(29.4 \mu \mathrm{M})$ and II $(31.1 \mu \mathrm{M})$. For vitamin C, group I showed lower plasma mean values $(3.84 \mu \mathrm{g} / \mathrm{ml})$ compared to groups II $(4.04 \mu \mathrm{g} / \mathrm{ml})$ and III $(5.61 \mu \mathrm{g} / \mathrm{ml})$. Vitamin A had the most similar mean levels between the three study groups, being for group I-4.76 $\mu \mathrm{M}$, group II-4.08 $\mu \mathrm{M}$ and group III-4.28 $\mu \mathrm{M}$. Antioxidant vitamins mean levels were statistically $(p=0.05)$ different between the study groups. For vitamin C, differences were observed between groups I and III. The mean values for vitamin A were different between diabetic patients with and without angiopathy and also between diabetics with angiopathy and nondiabetic subjects. The mean levels of vitamin E were significantly different between diabetic and nondiabetic subjects. The prevalence of hipovitaminose $\mathrm{C}$ was higher than $95 \%$ for all groups. For vitamin E, the percentage of subjects with low levels was for group I-60\%, group II-51\% and group III- $84 \%$. The prevalence of hipovitaminose A was insignificant in the study population. The prevalence of low levels of vitamins with anti-inflammatory proprieties does not seem to be related with the presence of diabetes or angiopathy. Vitamin $\mathrm{C}$ and $\mathrm{E}$ deficiency was high in diabetic and nondiabetic subjects. Supplementation with these two vitamins should be considered, especially in diabetic patients who have suppressed immunity and a higher risk to develop or aggravate infections.

This study was funded by PIC/IC/82957/2007 from "Fundação para a Ciência e a Tecnologia" (FCT) and Ana Valente is grateful for the PhD grant, number (SFRH/BD/16166/2004/5E4M) and the research grant, both supported by FCT. 